\title{
La réception des symphonies de Haydn à Paris. De nouvelles perspectives de recherche...
}

\section{Alexandre Dratwicki}

\section{(2) OpenEdition \\ 1 Journals}

\section{Édition électronique}

URL : https://journals.openedition.org/ahrf/2083

DOI : 10.4000/ahrf.2083

ISSN : 1952-403X

Éditeur :

Armand Colin, Société des études robespierristes

\section{Édition imprimée}

Date de publication : 1 juin 2005

Pagination : 83-104

ISSN : 0003-4436

\section{Référence électronique}

Alexandre Dratwicki, «La réception des symphonies de Haydn à Paris. De nouvelles perspectives de recherche... ", Annales historiques de la Révolution française [En ligne], 340 | avril-juin 2005, mis en ligne le 15 juin 2008, consulté le 23 avril 2022. URL : http://journals.openedition.org/ahrf/2083 ; DOI :

https://doi.org/10.4000/ahrf.2083

Ce document a été généré automatiquement le 23 avril 2022.

Tous droits réservés 


\title{
La réception des symphonies de Haydn à Paris. De nouvelles perspectives de recherche...
}

\author{
Alexandre Dratwicki
}

"Mozart est, après Haydn, le premier de tous les grands compositeurs. "

Tablettes de Polymnie, 5 novembre 1810

1 Cet avis d'un périodique du Premier Empire prête aujourd'hui à sourire. Il rappelle pourtant très justement qu'Haydn connut seul, de son vivant, une gloire incontestable sur le territoire français alors qu'il fallut à Mozart beaucoup de temps pour s'imposer dans les programmes musicaux. "C'est le privilège du génie de Haydn de faire l'ouverture de tous les concerts " ${ }^{2}$ note le Courrier de l'Europe en 1810 . Cet engouement apparaît bien plus tôt, dans les dernières années de l'Ancien Régime, enjambant sans obstacle la période révolutionnaire pour s'épanouir pleinement sous l'Empire. Il semble qu'en aucune façon la musique de Haydn ne fut jugée " aristocratique » au moment des événements de 1789, à une époque où l'on bannissait pourtant de la scène lyrique les chefs-d'œuvre de Gluck trop conformes aux goûts de l'ancienne monarchie. Quant à dater précisément l'introduction des symphonies du compositeur en France, François Lesure conforte l'avis de Lionel de La Laurencie ${ }^{3}$ : « 1764 connaît la première édition d'une symphonie d'Haydn, à Paris chez Venier. Mais il semble que Lyon ait eu le privilège d'entendre avant Paris sa première symphonie. Alors que le Concert Spirituel affiche en 1773 l'exécution d'une telle œuvre, l'Académie du Concert de Lyon interpréta le 29 avril 1772 une "symphonie à grand orchestre à quatre cors obligés de Hayden ", que l'on peut supposer avoir été les nos 13 ou 31 du Catalogue van Hoboken, bien que celles-ci ne paraissent pas avoir alors été éditées en France ${ }^{4}$.

2 La première preuve d'un succès avéré se manifeste par la commande du comte d'Ogny (1757-1790) de six symphonies destinées au répertoire du Concert de la Loge Olympique parisienne, société réputée dans toute l'Europe pour ses qualités d'exécution. En 1785 et 1786 , six chefs-d'œuvre voient ainsi le jour (symphonies $n^{\circ} 82$ à 87), inaugurant la série insurpassée des vingt-trois dernières symphonies du maître. Toutes ces 
compositions intéressèrent au premier chef les éditeurs français tant le public parisien montra une insatiable avidité à les entendre et les réentendre. La Symphonie en sol majeur dite « Militaire ", $\mathrm{n}^{\circ} 100$ du catalogue Hoboken ${ }^{5}$, incarne, de ce point de vue, un sommet de popularité. Chaque exécution - parfois presque quotidienne sous le Directoire et le Consulat - suscitait un enthousiasme exceptionnel, dont témoigne Johann Reichardt, de passage à Paris en 1803 :

"Cette musique à la turque enchantait le public! Chaque fois qu'elle retentissait avec un fracas formidable, l'assistance frémissait, les dames surtout tressaillant d'aise, applaudissaient avec enthousiasme en poussant des petits cris de plaisir. L'attitude de la partie féminine est amusante à observer dans les concerts parisiens. $»^{6}$

3 Reichardt, détaillant plus loin le pupitre des percussions (« une batterie de cymbales, de triangles, de timbales, de trompettes, une immense grosse caisse, posée très haut » [7]), atteste sans hésitation possible que l'œuvre était bien cette Symphonie "militaire " dont l'Andante ajoute à l'instrumentation classique deux clarinettes en ut, cymbales, triangle et grosse caisse. Plus souvent, malheureusement, il demeure impossible de savoir précisément quelles étaient les symphonies exécutées car les programmes ne donnent, la plupart du temps, aucun détail quant à la tonalité et au numéro d'opus de l'œuvre. Et quand bien même, les catalogues d'éditeurs ayant chacun adopté leur propre classement, il s'avère de toute façon très difficile d'assurer que tel ou tel morceau fut effectivement joué ${ }^{8}$. On lit parfois que l'orchestre interprètera «une nouvelle symphonie d'Haydn en ré ${ }^{9}$, « une nouvelle symphonie d'Haydn en sol ${ }^{10}$, ou encore une symphonie " en ut " ${ }^{11}$ ou " en sol mineur " ${ }^{12}$. Dans ce dernier cas, tout porte à croire qu'il s'agit de la Symphonie $n^{\circ} 83$ "La Poule», reconnaissable par cette tonalité peu usitée de sol mineur. Pour autant - dans bien des situations - rien ne permet une identification incontestable.

4 La réception des ouvrages de Haydn en France a déjà fait l'objet d'études musicologiques précises ${ }^{13}$. Le cas du Stabat Mater est aujourd'hui abondamment commenté. Mais en ce qui concerne les symphonies, leur succès n'a bien souvent été envisagé que sous l'angle convenu du concert public. Or ces concerts ne sont pas les seules opportunités d'entendre cette musique pour le Parisien de la Révolution: de récentes découvertes attestent d'un engouement beaucoup plus étendu pour les symphonies, leur exécution dépassant le cadre des sociétés de concerts publics pour infiltrer profondément d'autres aspects de la vie musicale.

Les symphonies de Haydn : œuvres modernes, savantes et difficiles

5 S'il est un point commun aux éloges de la presse française, c'est reconnaître en Haydn un compositeur aux effets neufs et originaux. «Le nec plus ultra du génie » ${ }^{14}$. Or dans les années 1780, la musique de l'avenir vient vraisemblablement de l'Italie. Aussi n'est-on guère étonné de voir le Concert Spirituel italianiser fréquemment les noms des artistes exécutés ou exécutants... «Les deux premiers morceaux de la symphonie "del signor Hayden" ont produit le plus grand effet ${ }^{15}$ assure le Journal de Paris. Personne ne doute alors que ce style musical soit l'aboutissement d'une longue maturation artistique dont on ne décèle que les prémices dans les ouvrages de jeunesse. Une comparaison flatteuse est justement proposée au public des Concerts de la rue de Cléry, à qui l'occasion est plusieurs fois donnée d'entendre, dans la même séance musicale, une des premières et une des dernières symphonies du maître. La Correspondance des Amateurs Musiciens rapporte par exemple: «le concert s'est ouvert par [la symphonie] en mi, l'une des anciennes d'Haydn; et la seconde partie par une des nouvelles. Il est quelquefois bon 
d'entendre des premières symphonies de ce compositeur à côté des dernières ; c'est un moyen de juger lequel est préférable ou de la science, qu'on trouve dans les unes, ou de la grâce et de l'originalité qui règnent dans les autres" ${ }^{16}$. Même confrontation quelques semaines plus tard, quand « deux symphonies d'Haydn, la première, une des anciennes, en $\mathrm{D}$ mineur, la seconde, une des douze dernières, en $\mathrm{G}$ majeur ${ }^{17}$, ont été exécutées avec la perfection accoutumée » ${ }^{18}$. À dire vrai, si après 1800 les opportunités d'écouter des symphonies de jeunesse se multiplient, ce n'est pas tant par souci didactique de confrontation, que pour éviter de lasser le public en ressassant trop souvent les mêmes ouvrages. Un journaliste insiste sur ce point après l'audition de deux symphonies «londoniennes " ${ }^{19}$ (notamment la Symphonie "militaire») : "Nous profiterons de cette circonstance pour inviter les directeurs du concert à faire entendre successivement plusieurs anciennes symphonies d'Haydn, qu'on n'a pas exécuté depuis long-temps; elles piqueront davantage la curiosité et exciteront plus d'intérêt que quelques autres plus modernes, qui ont été jouées peut-être trop souvent à la rue de Cléry ou au Conservatoire de musique " ${ }^{20}$. La saison du Concert des Amateurs, en 1805, attisa justement les foudres de la critique parce qu'au lieu de proposer des nouveautés, l'orchestre rejoua à trois occasions la même symphonie « en si bémol » ( ${ }^{\circ} 102$ ?) :

«L'usage de ne présenter au public que des symphonies avec lesquelles on l'a familiarisé depuis plusieurs années s'est maintenu jusqu'au sixième et dernier concert des amateurs. On n'a pas voulu leur faire entendre une seule des symphonies de Mozart, on n'a pas même voulu exécuter quelques-unes des symphonies d'Haydn qui sont moins connues, et on s'en est tenu invariablement à ne jouer que celles que les musiciens savoient par cœur. Aussi, peu à peu l'intérêt que devoient produire ces magnifiques compositions instrumentales a-t-il sensiblement diminué, quoique l'exécution fût toujours également parfaite. [...] La seconde symphonie, en si bémol, avoit même été déjà exécutée au quatrième ou au troisième concert de cette année. $»^{21}$

6 Paradoxalement, certains détails des premières symphonies semblent gêner l'auditeur de la fin du XVIIIe siècle. La structure " archaïque » en trois mouvements au lieu de quatre, par exemple, est à l'origine de manipulations artistiques douteuses...

« Nous ne terminerons point cet article sans parler d'un trio et d'un menuet de la composition de M. Viderker, qui ont été intercalés dans la première symphonie d'Haydn. Ces deux morceaux sont d'un effet charmant, et se soutiennent avec avantage dans le cadre où on les a placés : c'est le plus bel éloge que nous puissions en faire. " ${ }^{22}$

7 Ce type de modification ne concerne pas seulement les œuvres de jeunesse. Certaines des symphonies londoniennes connurent des changements similaires, dont plusieurs passent pour traditionnels: "Nous ne savons pas pourquoi on n'a jamais exécuté, à Paris, l'adagio en $f a$ de cette symphonie [en Si b M], et pourquoi l'on s'est toujours opiniâtré à y intercaler un andante d'une autre symphonie. On ne sauroit trop s'élever contre cette manie de faire des pasticci dans la musique instrumentale, à l'imitation des Italiens dans leurs opéras bouffons. Il faut respecter les grands maîtres et ne pas prétendre faire mieux qu'ils n'ont fait; assurément, si Haydn venoit à Paris, on n'oseroit pas jouer devant lui une symphonie dont on auroit pris l'allegro d'un côté, le minuetto de l'autre, \&c. ${ }^{23}$

8 L'obstination de quelques sociétés de concerts à n'exécuter que les dernières symphonies du maître s'explique peut-être par l'emploi systématique dans celles-ci d'un orchestre étoffé, avec flûtes, hautbois, clarinettes ${ }^{24}$, bassons, cors, trompettes, timbales et cordes, propre à faire valoir les grandes formations des principaux concerts 
parisiens. Le traitement "obligé » des instruments à vent y est particulièrement apprécié, qui rappelle l'engouement contemporain pour la symphonie concertante aux parties solistes si exubérantes ${ }^{25}$. Cette texture audacieuse, où dialoguent avec une égale importance tous les pupitres de l'orchestre, trouve en France un vrai terrain de prédilection. La presse annonce aussi souvent que possible ces «nouvelles symphonies " ${ }^{26}$ résolument modernes, pointant du doigt les instruments les plus sollicités. La Feuille du jour souligne par exemple l'exécution d'une "symphonie d'Haydn, dans laquelle les solos de basson, hautbois, flûte et clarinette seront exécutés par MM. Delcambre, Sallentin, Vanderlick et Lefebvre " ${ }^{27}$. Avant la Révolution, le Concert Spirituel fit entendre à plusieurs reprises une «symphonie avec cor et hautbois obligés » dont le Mercure de France signala la difficulté ${ }^{28}$. Mentionnons encore une «symphonie de M. Haydn, avec deux parties de cors obligés, exécutés par MM. Lebrun et Frédéric $"{ }^{29}$, donnée au Théâtre de Monsieur pour le concert spirituel du samedi 23 avril 1791. Il semble d'ailleurs que la première symphonie jouée à Paris reçut un accueil favorable justement grâce à son mouvement lent concertant. Le Mercure de France d'avril 1773 rapporte en tout cas qu'» il y eût, ce jour-là, deux symphonies au Concert, l'une au commencement (elle est de Toeschi), l'autre dans la seconde partie (celle-ci est de Haydn). Toutes deux ont été extrêmement applaudies, mais, entre autres, l'Andante de la seconde, dans lequel M. Rault exécuta un solo sur la flûte " ${ }^{30}$. À ce propos, La Laurencie ajoute: «ce détail du solo de flûte désigne nettement la symphonie en ré majeur [...] dont l'Adagio (et non l'Andante) porte la mention « Flauto solo » et quintette [...], indiquée comme écrite en $1764{ }^{31}$. On connaît certes le remarquable niveau technique des orchestres de la fin du XVIIIe siècle, notamment ceux du Théâtre Feydeau, du Concert de la rue de Cléry, de la rue de Grenelle, ou encore des élèves du Conservatoire, mais l'on sait moins qu'une qualité semblable (ou proche) exista aussi dans les petites salles parisiennes, autorisant sans problème l'exécution de ces symphonies : « Tous les [...] orchestres de Paris sont assez généralement bons, en raison de leur utilité pour les théâtres auxquels ils sont attachés; il seroit même difficile aujourd'hui de rencontrer, aux petits théâtres des boulevards, ou dans les bals publics, ces anciens orchestres discordans qui déchiroient autrefois les oreilles aussi impitoyablement. Il y a une amélioration telle que les pantomimes, les mélodrames, et jusqu'aux walses et aux contredanses, outre leurs compositeurs, pourroient aussi faire remarquer leurs virtuoses parmi leurs exécutans" ${ }^{32}$. Cuvres difficiles, mais gratifiantes, les symphonies de Haydn trouvèrent donc des interprètes à leur mesure sur le sol français.

Du coup, ce répertoire servit aussi la cause - certes moins louable - d'une concurrence forcenée entre les institutions de concerts, dont chacune voulait décrocher la palme de l'excellence. Le 25 février 1804, par exemple, les artistes de la rue de Grenelle font entendre une symphonie « en si bémol » ${ }^{33}$, "la même qui a été exécutée au précédent concert Cléry et nous avons cru y être encore. Cela prouve que la rivalité, bien entendue, est un aiguillon puissant pour arriver au bien. "On en vaut mieux quand on est comparé" ${ }^{34}$. Riposte immédiate en mars suivant : l'orchestre de la rue de Cléry exécute cette fois une symphonie «en mi mineur naturel ${ }^{35}$, [...] entendue au dernier concert de l'académie, rue de Grenelle ${ }^{36}$. En 1803 déjà, les élèves du Conservatoire avaient donné le 12 février « la même [symphonie] entendue au précédent Concert de la rue de Cléry, l'une des douze, faites pour le grand concert de Londres, et numérotée $91 »^{37}$. 
10 Ainsi se met en place une véritable tactique commerciale s'appuyant essentiellement sur les ouvrages de Haydn et justifiant leur place stratégique dans le déroulement des concerts parisiens. Elles en sont "l'ouverture obligée » ${ }^{38}$, précise sans équivoque la Gazette de Paris. Cette position enviable expliquerait même, selon un cercle vicieux dénoncé par le Journal de l'Empire, pourquoi «la symphonie d'Haydn est ordinairement ce qui fait le plus de plaisir dans un concert : c'est le morceau que l'on goûte le mieux, parce que l'attention est toute fraîche. Ceux qui ne viennent que pour sa musique ne perdraient presque rien, et quelquefois même gagneraient quelque chose, en s'en allant aussitôt après la symphonie; ils auraient beaucoup d'ennui et de fatigue de moins et remporteraient un sentiment délicieux de la plus agréable harmonie; ils n'auraient point entendu de voix humaines dont les agréments sont toujours mêlés d'imperfection, mais ils auraient entendu des violons, des flûtes, des hautbois, des clarinettes qui chantent fort bien, dont les voix sont belles, toujours justes et sans défaut ${ }^{39}$. Rares sont les concerts, entre 1790 et 1810 , qui dérogeront à cet impératif. Au point que le Journal des Débats s'étonne, lors d'une prestation au bénéfice de Mme Grassini en 1801, que l'orchestre ait commencé «non par une symphonie d'Haydn, qui dans tous nos concerts étoit depuis longtemps un début officiel, mais par une ouverture de Mozart " ${ }^{40}$... L'enthousiasme est parfois poussé plus loin, et nombre de soirées musicales font entendre jusqu'à deux symphonies dans leur entier, en début de chaque partie. Peut-être justement afin d'éviter que l'assistance ne quitte la salle après l'exécution de la première ou à l'entracte... Les Concerts Feydeau eurent très tôt recours à cette stratégie (par exemple les 20 et 22 avril $\left.1791{ }^{\left[{ }^{4}\right]}\right]$, ou plus tard le 12 juin [ $\left.{ }^{42}\right]$ de la même année), et Johann Friedrich Reichardt décrit une organisation encore similaire au concert de la rue de Cléry en décembre $1802{ }^{43}$ et janvier 1803 : «l'exécution des symphonies a été admirable, comme à l'ordinaire. L'une et l'autre de celles que nous avons entendues font partie des douze dernières qu'Haydn a composées pour le grand concert de Londres $"{ }^{44}$. Cette structuration rigoureusement similaire d'un programme de concert à l'autre laisse apparaitre une sorte d'» académisme ", au sens le plus fort du terme. Car justement - consécration suprême les symphonies de Haydn servent sous l'Empire à introduire les séances solennelles publiques de l'Académie des Beaux-Arts. À mi-chemin entre le concert et la conférence, ces séances de l'Institut font alterner discours et exécutions musicales. On y entend notamment la cantate du prix de Rome récompensée dans l'année. Et tandis que les orateurs en appellent aux meilleures compositions devant servir de modèle au jeune lauréat, l'orchestre exécute en " ouverture » à la cantate l'une ou l'autre des grandes symphonies de Haydn ${ }^{45}$.

11 Un mot encore sur une littérature foisonnante : celle de la transcription instrumentale pour ensemble plus ou moins réduit. Encouragé par l'essor formidable de l'édition musicale parisienne à la fin du XVIII ${ }^{\mathrm{e}}$ siècle, l'arrangement devint rapidement l'aliment musical de base pour tout amateur cultivant - volontairement ou pas - une sociabilité artistique d'Ancien Régime. Il n'est pas possible d'aborder ici en détail l'imposante quantité des pièces de Haydn publiée à Paris pendant la Révolution et l'Empire (essentiellement les quatuors à cordes et trios avec clavier). Seul importe de préciser que les symphonies ne sont pas restées longtemps étrangères au répertoire de la musique de chambre. Elles prêtèrent à de nombreuses transcriptions, notamment de la main de Clementi (pour pianoforte, flûte, violon et violoncelle ad libitum) et du violoniste anglais Salomon, lequel réduisit les douze dernières symphonies du maitre 
pour flûte, quatuor à cordes et clavier ad libitum (ce dernier, à vrai dire, se contentant de réaliser une harmonisation de la partie de violoncelle, exactement dans l'esprit d'un continuo baroque). Elles parurent notamment chez Carli et Gambaro dans les années $1815^{46}$. Moins connus, parce que moins édités, furent les arrangements pour orchestres d'instruments à vent que sollicitèrent tant les manifestations révolutionnaires, et dont tous les jardins publics retentissaient une fois le printemps revenu: Frascati, Tivoli, L'Élysée attiraient une partie de leur clientèle en annonçant ces grands «concerts d'harmonie ", pas si exceptionnels, à vrai dire, que les annonces ne le suggèrent parfois. Malheureusement, les programmes de la presse poussent rarement dans le détail. Il n'empêche, Haydn y paraît souvent à l'honneur, comme l'atteste une mention du Courrier des Spectacles à l'occasion d'une fête au Pavillon de Hanovre, le 1er septembre 1799 : un "air de Haydn " ${ }^{47}$ fut dirigé par Solère, à la tête d'un orchestre d'instruments à vent visiblement très étoffé. Entendu au sens de "mélodie connue ", cet air n'était probablement rien d'autre qu'un mouvement détaché de symphonie.

Hors du concert...

12 Les commentateurs actuels disent trop rarement à quel point l'expansion des symphonies de Haydn à Paris se fit également hors des structures habituelles du concert public. À peu près tous les théâtres disposant d'un orchestre allaient promouvoir cette musique, sinon par des exécutions irréprochables, en tout cas avec un zèle remarquable entre 1780 et 1815 . La diffusion de ce répertoire fut d'autant plus rapide que la Révolution avait instauré la libre création d'entreprises de spectacles, autorisant enfin ce que l'Ancien Régime avait trop étouffé : la concurrence dont dépend «le perfectionnement de l'art " ${ }^{48}$. Aussitôt, l'éclosion de dizaines de salles aux projets artistiques plus ou moins ambitieux assura l'organisation de multiples phalanges instrumentales.

13 Une habitude déjà ancienne voulait que les musiciens occupent les entractes des comédies et tragédies parlées avec des pièces instrumentales variées. Les archives de la Comédie-Française possèdent le détail d'un " état des Simphonies " ${ }^{49}$ achetées pour cet usage en 1788. Haydn y figure en première place, avec six de ses compositions, aux côtés de Rigel (6), Guénin (3), Cambini (3), Ragué (3), Barrière (3) et Janson (3). Selon Stendhal, l'exécution de certaines «symphonies à grand orchestre » de Haydn étaient d'ailleurs idéales dans les théâtres "à cause du grand nombre d'instruments nécessaires $"{ }^{50} \ldots$.. Mais la question complexe des effectifs reste difficile à éclaircir, car peu d'institutions musicales possédaient des ensembles instrumentaux avec pupitres de vents au complet. À titre d'exemple, pour l'année 1805, il « manque » - selon le schéma admis de l'orchestre classique - 1 flûte, 2 hautbois et 1 basson à la Comédie-Française ("Théâtre français »); 2 flûtes, 2 hautbois et 1 basson au Théâtre du Vaudeville; 2 hautbois et 1 basson au Théâtre de l'Ambigu-Comique ; 1 flûte, 2 hautbois, 1 clarinette et 1 basson au Théâtre des Jeunes Artistes... De toutes les salles parisiennes, seul l'Opéra

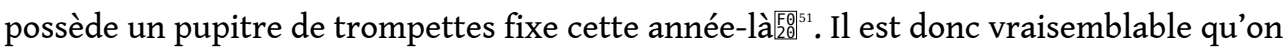
exécutait certaines compositions en omettant les parties les moins indispensables, ce que suggère le Journal de Paris: «La séance [au Conservatoire] a commencé par une symphonie de Haydn, symphonie qu'on entend tous les jours et partout, qu'écorche l'orchestre de la Comédie-Française et voire même celui du Café des Etrangers au Palais-Royal " ${ }^{52}$. Or, sans aucun doute, l'orchestre du Café des Étrangers devait paraître bien modeste comparé à la formation colossale du Conservatoire. 
Dans ce contexte post-révolutionnaire où l'émulation rend l'expérimentation journalière, les ouvrages de Haydn ne se contentent pas de tenir lieu d'entractes agréables et de musique "d'ambiance». La technique du pot-pourri - pastiches, collages et parodies - contaminant une grande quantité de spectacles, l'aubaine d'une musique aussi célèbre retint vite l'attention des dramaturges et compilateurs en tous genres. "L'Enfant Prodigue, mélodrame en quatre actes et à grand spectacle, mêlé de pantomimes, danses et combats ; paroles de J. G. Cuvelier et J. B. Hapdé. La musique est tirée des œuvres de Bocherini, Haydn, Pleyel, Kreutzer, et plusieurs autres auteurs célèbres" ${ }^{53}$, annonce le Courrier des Spectacles du 14 janvier 1814. Voilà donc les symphonies de Haydn étroitement liées à une action dramatique, et parfois même chargées de l'introduire. Reichardt, assistant à une représentation de Roland de Monglace (mélodrame en quatre actes de Loaisel-Tréogate) au Théâtre de la Porte SaintMartin, apprécie d'y entendre un "orchestre excellent qui avait parfaitement interprété une symphonie de Haydn, [...] en guise d'introduction " ${ }^{54}$. Quelques années plus tôt, le compte rendu de la création des Mystères d'Isis ${ }^{55}$ à l'Opéra n'omet pas de décrire lui non plus «la belle introduction du quatrième acte [...], au moment où Bochoris une lampe à la main et tremblant de frayeur descend dans le souterrain, [c']est le commencement d'une Symphonie d'Haydn, mais si bien adaptée à la scène qu'elle sembleroit avoir été composée exprès ${ }^{56}$. Il s'agit là de l'introduction lente de la Symphonie $n^{\circ} 103$ en Mi b M «Roulement de timbales »... Des expériences variées acclimatent ainsi la musique du compositeur à leurs propres exigences dramatiques, avec - il faut l'avouer - plus ou moins de bonheur. Citons l'anecdote relevée par François Lesure qui rappelle "l'utilisation du second mouvement de la symphonie "L’Impériale", transformé en romance avec les paroles, "je ne vous disais point: j'aime". Cet air fut inséré dans L'Inconséquente ou Le Fat dupé, comédie en un acte et en prose par M. Monnet, comédien, donné au Théâtre des Variétés au Palais Royal le 20 août 1787 et dont le livret, imprimé par Cailleau (avec la musique de l'air) est conservé à la bibliothèque du Conservatoire. La romance est chantée par l'un des quatre personnages de la pièce, l'abbé, qui s'accompagne à la guitare. Cet air fut ensuite repris dans diverses anthologies avec le titre "Ariette du fat dupé" $\gg{ }^{57}$. Le succès de cet arrangement devint assez populaire pour que l'incipit de l'air renvoie directement à la symphonie en question. On lit par exemple dans le Courrier des Spectacles du 10 avril 1802: «Le concert qui a eu lieu le 20 n'a pas offert l'ensemble ordinaire; on a cependant beaucoup applaudi dans la simphonie d'Haydn en ré le charmant andante : je ne vous dirai pas j'aime $»{ }^{58}$.

15 Autre exemple atypique, celui d'un chœur avec solos de ténor écrit sur le motif du mouvement lent de la Symphonie "Militaire»: «L'Andante [...] offre une petite particularité anecdotique qui honore son auteur. Il est si régulier dans son motif, dans son rythme, dans ses détails, qu'il a fourni le sujet d'un petit poëme, mêlé de chœurs, et de solos, qui a été exécuté avec applaudissemens dans le concert de Garat au théâtre Feydeau $»^{59}$.

16 Mais parmi toutes les bonnes fortunes du répertoire symphonique de Haydn en France, la plus éclatante - la plus originale aussi - fut sans conteste son intégration aux structures chorégraphiques du nouveau ballet-pantomime. Pour Castil-Blaze, «les belles symphonies de Haydn, les ouvertures, la musique dramatique, les concertos, les duos de violons, les sonates, les romances, les barcarolles, toutes ces pièces diverses servent à la composition des ballets d'action ${ }^{60} . .$. 
Un support chorégraphique idéal

« [Gardel vient de produire dans son dernier ballet] une preuve nouvelle [de son talent] dans l'application qu'il vient de faire de son art, de l'un des plus agréables et des plus savants andante d'Haydn. L'alternative des piano et des forte, qui font le charme de ce morceau sont rendus tour-à-tour par la citoyenne Gardel et la citoyenne Pérignon, chacune dans le genre qui lui est propre, pendant que la partie si originale des basses, leurs variations et jusqu'à leurs rentrées, sont figurées par les Cnes Milliere et Louise. Cet ensemble est admirable surtout par la précision et les nuances particulières de ce genre, dont chaque sujet colore son jeu. Légèreté, rapidité, finesse dans celui de la citoyenne Gardel ; force, à-plomb, énergie dans celui de la cit. Pérignon ; volubilité, symétrie et exactitude dans celui des cit. Louise et Milliere. Tels sont les caractères distinctifs des parties qui forment l'ensemble de ce charmant ballet ; il a ravi tous les spectateurs en général. " ${ }^{61}$

La Fête de Mirza, ballet-pantomime de Gardel et Gossec créé en 1780, fut le premier ouvrage chorégraphique français à proposer un collage d'airs connus en guise de trame musicale expressément narrative ${ }^{62}$. L'idée faisant école, la période révolutionnaire vit bientôt éclore les trois ballets les plus célèbres du répertoire de l'Opéra jusque sous la Restauration: en 1790, Télémaque dans l'île de Calypso, puis Psyché (chorégraphie de Gardel et musique de Miller); en 1793, Le Jugement de Pâris, toujours élaboré par Gardel, mais sur une musique arrangée par Méhul. Outre leur succès immédiat et prolongé, ces trois ouvrages intéressent directement par l'emploi systématique de mouvements symphoniques de Haydn. Si Télémaque et Psyché n'exploitent ce répertoire que de façon parcimonieuse (deux mouvements isolés dans chaque ballet), Le Jugement de Pâris, par sa légère postériorité, atteste de l'enthousiasme rapide pour le procédé. On y entend successivement, disséminés tout au long des trois actes, les sept extraits suivants :

- Symphonie $\mathrm{n}^{\circ} 44$ « Funèbre », 3e mouvement « Adagio » (1765)

- -Symphonie $\mathrm{n}^{\circ} 31$ «Appel de cor», 4e mouvement «Finale, Moderato molto »

(1765)

- Symphonie $\mathrm{n}^{\circ}$ 91, 3e mouvement « Menuetto » (1788)

- Symphonie $\mathrm{n}^{\circ}$ 91, 2e mouvement « Andante» (1788)

- -Symphonie n॰ 49 «La Passion », 2e mouvement « Allegro di molto » (1768)

- Symphonie n' 82 « L'Ours », 2e mouvement « Allegretto » (1786)

- Symphonie $n^{\circ} 73$ « La Chasse », 4e mouvement, « Presto » (c.1781)

Volontairement ou pas, cette diversité satisfait à deux des paramètres observés dans le cadre des concerts publics. D'une part, l'alternance entre compositions de jeunesse et de maturité, de l'autre, l'emploi opportun d'instruments solistes. Choisi certainement en fonction de ce second critère, le final de la Symphonie $n^{\circ} 31$ s'organise selon la coupe $\mathrm{du}$ thème et variations, ici pour hautbois, violoncelle, flûte, cors, violon et contrebasse solistes. Dans la quatrième variation en particulier, quatre (!) cors se livrent à des prodiges de virtuosité. La Symphonie $n^{\circ} 91$ s'attache quant à elle à faire valoir le premier basson de l'orchestre. D'abord dans la variation initiale de l'Andante, puis dans le trio du menuet, au caractère proche du ländler. Ces mouvements de symphonies, chorégraphiés par les meilleurs artistes de l'Opéra, sont recopiés intégralement dans les partitions des ballets, sans doute à partir des premières éditions parisiennes disponibles. On note à chaque fois un respect scrupuleux des détails d'instrumentation: le ballet Alexandre chez Apelles de Catel (1808) emprunte ainsi à la Symphonie $n^{\circ} 100$ "Militaire"son mouvement lent, sans omettre sa percussion «turque » caractéristique. Les emprunts à Haydn occupent souvent une place de choix aussi stratégique que l'ouverture de rideau sur un nouveau décor (au troisième acte de Télémaque de Miller par exemple) ou la péroraison d'un divertissement rassemblant tous les danseurs du ballet (final de l'acte I 
du même Télémaque, ou dernier final du Jugement de Pâris de Méhul, juxtaposant des extraits des symphonies $\mathrm{n}^{\circ} 82$ "L'Ours» et 73 "La Chasse»). Cette présence à des moments clefs du spectacle - particulièrement en début d'acte - renvoie directement à l'agencement des programmes de concerts publics contemporains où le même privilège a déjà été souligné.

La proximité entre ballets-pantomimes autonomes et divertissements de tragédies lyriques (mêmes danseurs, orchestre, chorégraphe, costumes, décors, espace...) induit une quantité de réflexes musicaux similaires, à commencer par le choix des œuvres destinées à être dansées. Les emprunts à Haydn dépassent là encore toute attente puisque non seulement certains mouvements de symphonies complètent les ballets des tragédies modernes, mais surtout elles s'intègrent a posteriori aux reprises d'opéras plus anciens. Les célèbres ouvrages de Gluck sont « rehaussés » - selon l'avis de l'époque - de pas ajoutés sur une musique de Haydn. Au tout début du XIX ${ }^{e}$ siècle en particulier, une nouvelle production d'Armide suggère « des ballets charmans [qui] complètent tous les plaisirs. [...] On a substitué un air de ballet d'Iphigénie en Aulide, un autre d'Echo et Narcisse. [...] Mais ce qui, aux yeux des amateurs les plus difficiles d'un art aussi séduisant, ne peut manquer d'ajouter beaucoup à la réputation du cit. Gardel, ce sont les dessins nouveaux qu'il vient d'établir sur la belle romance d'Haydn. La gradation, l'enchaînement et la variété des groupes y sont enchanteurs. Tout y est simple, et tout y produit des tableaux qui surprennent les sens et les captivent en 1801, l'adaptation française de La Flûte enchantée de Mozart recourt au même procédé :

"C'est sur un des plus agréables andante d'Haydn que Mlle Saulnier danse avec la précision, le moëleux et l'aplomb qu'on ne cesse d'admirer en elle ; c'est encore sur la meilleure musique d'Haydn, de Pleyel et de Sacchini, que mesdemoiselles Clotilde et Chameroy, le cit. Aumer et mademoiselle Felicité et, dans un pas de quatre parfaitement ordonné, les citoyens Branchu, Beaulieu et mesdemoiselles Louise et Millière se disputent les suffrages des spectateurs. " "

Parmi les dizaines d'exemples à signaler dans les ouvrages chorégraphiques composés entre 1790 et $1830{ }^{65}$, mentionnons celui du ballet déjà cité Alexandre chez Apelles. Outre l'Andante de la centième symphonie, Catel introduit le menuet de la Symphonie $n^{\circ} 99$ en Mi $b \mathrm{M}$, entendu dans son entier pour conclure l'acte I. Le tableau suivant emprunte à Mozart sa grande Symphonie en sol mineur (KV 550) dont il extrait le mouvement initial (avec la première version de l'instrumentation, sans les clarinettes). Le déroulement s'interrompt curieusement au moment de la reprise d'exposition, juste avant que ne commence la zone de développement. Est alors joué le menuet de la Symphonie $n^{\circ} 99$ de Haydn, déjà entendue à l'acte $\mathrm{I}$, dont la première section - la partie « $\mathrm{A}$ » du menuet réapparaît presque intégralement. Nouvel arrêt brusque et voici l'auditeur replongé dans la symphonie de Mozart. Non pas là où il l'avait laissé (au début du développement), mais bien après, lors du « pont » entre les deux groupes thématiques, maintenus au ton principal de sol mineur comme l'exige la réexposition traditionnelle de la forme sonate. Encore interrompu quelques mesures avant la fin, Mozart cède à nouveau la place à Haydn, dont l'orchestre exécute en partie le trio du menuet précédent... Cette succession de ruptures peut être clarifiée ainsi :

21 Le public de l'époque salua la prouesse et l'ingéniosité de Catel. Sauf peut-être certains étrangers pour lesquels la spécificité du ballet français en "pot-pourri » faisait, à l'inverse, figure de barbarisme. L'Allemand Reichardt se lamentait dès 1803 : 
«Ce qui contribue à diminuer pour moi l'attrait des ballets [français], c'est leur musique, composée d'airs ramassés dans tous les quatuors, symphonies, sonates ou opéras connus. Les nobles motifs d'Haydn sont accouplés aux plus plates rapsodies et le tout s'exécute sur un rythme qui s'accélère ou se ralentit suivant les seuls caprices de la ballerine, sans que l'on s'occupe d'habiller d'un peu d'harmonie cette mosaïque étrange, afin d'en dissimuler les reprises manquées et les bigarrures choquantes. Et dire que cela se passe à Paris où Rameau avait porté si loin la perfection de la musique des ballets! ${ }^{66}$

Mais il va sans dire que la construction élaborée par Catel dans Alexandre chez Apelles atteste d'un projet dramaturgique autrement plus ambitieux que le simple collage. Les multiples interruptions de la musique, d'autant mieux senties qu'elles s'effectuent justement sur des ouvrages connus, correspondent au conflit des sentiments du roi Alexandre, à la fois curieux de connaître son rival, et bouleversé par l'amour de Campaspe pour un autre homme. L'étude du livret et le souvenir de l'expression utilisée par Momigny pour caractériser le premier mouvement de la symphonie de Mozart («Allegro molto. Motif d'une douleur exaltée " $\left.{ }^{6}\right]$ ) éclairent le choix du compositeur. Force mentions insistent sur les sentiments des personnages en scène, souvent fugitifs ou paradoxaux. En moins d'une page, on relève: "l'air agité d'Ephestion », «l'air chagrin de Campaspe", "Alexandre paraît inquiet", "les princesses ne peuvent dissimuler leur inquiétude ", "Campaspe ne peut s'empêcher de montrer le comble de l'accablement", "Alexandre, avec un vif empressement", "le roi a peine à se posséder ", "l'air sombre et agité d'Apelles ». À raison, le compositeur juge efficace l'alternance brutale entre la symphonie de Mozart et celle de Haydn pour dépeindre ce climat d'angoisse et de surprises. Mais, en parfait théoricien, Catel structure la dimension formelle de l'ensemble avec une grande cohérence musicale. On peut ainsi envisager le menuet de Haydn - en Mi b majeur - comme substitut au développement de la symphonie en sol mineur de Mozart, et le brillant trio en ut majeur comme une vaste coda à l'ensemble. La tension harmonique de la forme sonate se résout idéalement par la réexposition du second thème de la symphonie de Mozart dans le ton principal. Il y a donc drame scénique en même temps que drame musical. Cette convergence des effets cherche à peindre au mieux l'expression d'émotions complémentaires (crainte, curiosité, douleur, colère).

Alors se dessinent les qualités sous-jacentes de la musique de Haydn, à la fois enrichie par une confrontation avec les arts visuels du spectacle et dotée intrinsèquement d'une faculté descriptive exceptionnelle. Stendhal avoue : «J'ai souvent pensé que l'effet des symphonies de Haydn et de Mozart s'augmenteraient beaucoup si on les jouait dans l'orchestre d'un théâtre, et si, pendant leur durée, des décorations excellentes et analogues à la pensée principale des différents morceaux se succédaient sur le théâtre. Une belle décoration représentant une mer calme et un ciel immense et pur, augmenterait, ce me semble, l'effet de tel andante de Haydn qui peint une heureuse tranquillité. [...] De tels tableaux sur le théâtre seraient un excellent commentaire aux symphonies de Haydn, et les fixeraient à jamais dans la mémoire " ${ }^{68}$. Le balletpantomime français dépassa tous ses espoirs, ajoutant aux décors souhaités la matérialisation d'une action chorégraphique.

Une musique porteuse de sens... ou les prémices d'un « romantisme » musical

$\mathrm{Au}$ tournant des $\mathrm{XVIII}^{\mathrm{e}}$ et $\mathrm{XIX}^{\mathrm{e}}$ siècles, la musique instrumentale devient un vecteur d'expression insoupçonné jusqu'alors ${ }^{\text {ต. }}$. Pour certains, comme Grétry, elle est désormais apte à surpasser la musique scénique dans l'évocation d'un sentiment. $\mathrm{Ne}$ 
conseille-t-il pas lui-même de « regarder les œuvres innombrables de Haydn comme un vaste dictionnaire où [le compositeur dramatique] peut sans scrupule puiser des matériaux $"{ }^{70}$ ? Le résultat est celui d'une musique instrumentale savante passée au crible d'une analyse narrative par les compositeurs et les commentateurs du moment :

«Le célèbre Haydn a créé un genre nouveau dans ses symphonies; leur conception neuve, hardie et pathétique a élevé la musique instrumentale au rang de la musique dramatique, en distribuant si bien les rôles de chaque instrument qu'on les voit tous concourir au développement d'une action conduite et menée à son terme avec un art admirable; c'est pourquoi la musique d'Haydn est employée avec tant de succès dans la pantomime, véritable épreuve pour les compositions instrumentales, puisqu'il n'est de bonne musique que celle qui forme un tableau dans l'imagination ou qui fait naître un sentiment dans le cœur. " ${ }^{n}$

Plus encore, la musique doit être "parlante». "Quel auteur [...] n'a été saisi d'admiration en écoutant les belles symphonies d'Haydn! Cent fois je leur ai prêté les paroles qu'elles semblent demander. Eh! pourquoi ne pas les leur donner? " ${ }^{72}$. Si l'idée n'eut pas de véritable suite dans les opéras, le ballet-pantomime, au contraire, l'acclimata complètement. Cette perception essentiellement théâtrale de la musique instrumentale ira jusqu'à forger une "école» de pensée - oserait-on parler d'» analyse » ? - qui verra en chaque composition un monologue pathétique, un duo langoureux ou un brillant quatuor d'opéra. Cette époque affublera d'ailleurs nombre d'œuvres d'un titre souvent anecdotique... et justement quantité de symphonies de Haydn.

De fait, le public du concert ne pouvait plus faire abstraction des images et des actions scéniques que les spectacles divers avaient associées à certaines de ces partitions. Le souvenir transmettait aux yeux le plaisir des oreilles. La fameuse Symphonie "militaire " en souffrit - ou y gagna - peut-être davantage que n'importe quelle autre : «L'andante [...] offre à l'imagination étonnée un vaste tableau qui semble représenter toutes les richesses, tout le brillant éclat d'une pompe orientale. C'est un magnifique sultan assis sur son trône de gloire, entouré de tous les plaisirs, enivré de tous les délices ; c'est, en un mot, ce beau idéal qui n'appartient qu'au génie, dont la définition n'est dans aucune langue. Le grand opéra doit s'emparer de ce sujet. La féerie, les illusions, les enchantemens, voilà son domaine, et le succès n'en sauroit être douteux s'il est placé avec l'intelligence et le soin dont il est digne $"{ }^{73}$. Les descriptions parfois saugrenues dans lesquelles la presse allait se fourvoyer espéraient à l'inverse affiner la perception de ces chefs-d'œuvre... oubliant finalement la conception d'abord théorique de la musique instrumentale. Développements thématiques ou originalités harmoniques sont passés sous silence, même si l'on devine - affleurant le commentaire - combien la subjectivité poétique des critiques s'appuie sur des détails de facture bel et bien techniques ${ }^{74}$ :

«La symphonie en ré, de Haydn, op. 91, a fait l'ouverture [du concert]. Elle commence par un adagio admiratif, en mineur, d'un style sévère et belliqueux. Des plaintes qu'exhalent plusieurs interlocuteurs se font entendre çà et là. Ce sont des malheureux qui semblent implorer la clémence des dieux; leurs gémissements les touchent enfin, et le brillant allegro, majeur, qui suit, comme un ange de consolation, change cette scène de douleurs et de peines en une fête riante; tout le reste du morceau soutient le même caractère. L'andante suivant me paroit être une action de graces à la divinité. Il tient un peu de la couleur et du style romantique, mais il n'est pas moins grand et sublime. La fête recommence au menuet. C'est une danse champêtre sous un berceau de fleurs. Les hautbois, les cornemuses semblent inviter les pastoureaux et les bergères à la walse folâtre et voluptueuse. Le finale 
spiritoso annonce un chœur général d'allégresse et de contentement. Les groupes divers qui le composent sont très-distincts. Les âges, les conditions, tout est caractérisé par le grand maître qui sait tout peindre. Je m'arrête et m'incline devant toi, sublime Haydn !... A toi seul appartient l'analyse des mystères de ton génie. ${ }^{75}$

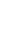
méthodes d'instrument prônées par le nouveau Conservatoire. Celle de Cambini pour violon ${ }^{76}$, parue chez Naderman en 1800, propose d'étonnantes saillies "poéticolittéraires » dont l'une d'elles nous concerne directement puisqu'elle porte sur le thème de l'andante de la Symphonie $n^{\circ} 53$ en RE M, dite "L'Impériale ». Souhaitant développer chez l'élève un sens prononcé du phrasé, Cambini attribue d'emblée un caractère extramusical à la mélodie initiale : "son expression est la naïveté, la candeur, l'innocence d'une bergère qui reproche à son amant de l'avoir trahie " ". Lorsque, par la suite, le pédagogue s'intéresse aux détails des doigtés et des coups d'archet, un même degré d'approfondissement est proposé parallèlement à l'»intrigue théâtrale »: «Vous voulez [atteindre le but de l'auteur], prenez-vous y de la manière suivante : Pénétrezvous d'abord du sentiment naïf et tendre qu'une jolie villageoise encore vierge éprouve en reprochant à son amoureux l'infidélité qu'elle méritait si peu. Supposez-lui un caractère encore plus naîf que celui de Colette dans Le Devin du village. Elle ne connaît pas le dépit, elle n'écoute que sa tendresse, elle ne dit que les paroles suivantes : Quoi! Tu peux m'être infidèle! Qui t'aimera plus que moi! Si je te parais moins belle, Mon cœur n'est-il rien pour toi ?... ou quelque chose de semblable mais mieux exprimé. [...] Voilà, j'en suis sûr, ce qu'a voulu l'auteur, intéresser, attendrir, affecter le spectateur. Hélas! que ceux qui ne regardent la musique instrumentale que comme un vain bruit n'ont-ils comme moi entendu exécuter les quatuors de Boccherini, de Haydn et de quelques autres maîtres célèbres par Manfredi, Boccherini, Nardini et moi, qui étais trop heureux de faire l'alto. Je suis bien sûr qu'ils tiendraient un autre langage. Ils avoueraient que l'art dramatique a toujours inspiré ces grands maitres même dans les ouvrages où elle ne peut se montrer aux yeux ${ }^{78}$. Yves Gérard, dans un commentaire de cette méthode de Cambini, rappelle la suppression par Haydn lui-même d'éléments trop naïvement descriptifs dans l'arrangement pour piano de son oratorio Les Saisons, et doute que le compositeur « ait vu autant de significations dans son modeste morceau de "musique pure", et même qu'il les eût acceptées sans protester " ${ }^{79}$. Cela dit, si Haydn réfute ordinairement toute signification poussée dans sa musique instrumentale, il ne profite pas moins de l'engouement général qu'elle suscite. Aussi réemploie-t-il volontairement l'andante de sa Symphonie $n^{\circ} 94$ "La Surprise » dans un air de baryton des Saisons, au moment où le laboureur est représenté aux champs, fredonnant une chanson populaire en poussant sa charrue. "Van Swieten blâma l'air des Saisons où le laboureur [...] siffle la mélodie de l'andante avec le coup de timbales. Il voulut persuader Haydn de le remplacer par une chanson tirée d'un opéra vraiment populaire et en cita lui-même deux ou trois. Haydn se montra offensé par une telle impudence et répondit avec sa fierté habituelle : "Je n'y changerai rien, mon andante est aussi bon et aussi connu que n'importe quelle chanson dans ces opéras" $"$ " .

Cette recherche de sens, cette nécessité de stimuler l'imagination quitte à détourner la finalité première des compositions symphoniques de Haydn (éventuellement par une 
débauche de moyens scéniques) témoignent d'un profond bouleversement de l'art musical au tournant des $\mathrm{XVIII}^{\mathrm{e}}$ et $\mathrm{XIX}^{\mathrm{e}}$ siècles et annoncent les prémices d'un romantisme ouvertement "expressif ». Ce dernier s'appuie finalement autant sur un répertoire en pleine émergence (Schubert, Weber, Spohr, Onslow...), que sur des œuvres plus anciennes qu'il dévoie de ses fonctions premières. Un peu comme si l'idée précédait l'objet, comme si une nouvelle façon de penser la musique ne pouvait, dans un premier temps, s'attacher à autre chose qu'à un support en décalage avec ce qu'on souhaite lui faire dire. Le besoin de modifier cet objet musical, avant l'apparition d'un autre plus satisfaisant, engendre alors les curieuses métamorphoses observées. De plus en plus souvent, après les années 1815 , les programmes de concerts morcèlent les exécutions des symphonies de Haydn. L'extraction hors du moule classique en quatre mouvements affirme l'autonomie de caractère de certaines pages et rejoint les préoccupations esthétiques de toute cette littérature de "l'instant », pièces de genres pour orchestre ou piano aux appellations si diverses («nocturne", "romance sans parole », « scènes pittoresques »...). L'exemple des procédés de fragmentation appliqués au menuet de la Symphonie $n^{\circ} 99$ dans le ballet de Catel est l'un des plus représentatifs de la naissance d'un goût nouveau. L'édition trahit aussi ce bouleversement de la pensée, lorsque paraissent séparément dans les années 1820 Douze Adagio ou Andante tirés des simphonies d'Haydn arrangés pour piano avec accompagnement de violon (et basse ad libitum) ${ }^{81}$.

31 Mais l'auditeur d'aujourd'hui a bien du mal à concevoir la musique de Haydn comme «romantique ». Stendhal, lui, n'hésite pourtant pas à écrire: «le caractère de la musique instrumentale de notre compositeur est d'être pleine d'une imagination romantique. C'est en vain qu'on y chercherait la mesure racinienne; c'est plutôt l'Arioste ou Shakespeare, et c'est ce qui fait que je ne comprends pas encore le succès de Haydn en France ${ }^{82}$. Paradoxe que tout cela ? Pas vraiment, car il est bien normal de ne pas trouver dans la musique de Haydn une description précise d'objets ou d'histoires qu'elle n'a jamais prétendu décrire! Période nécessairement trouble d'une “ révolution " artistique en cours...

\section{NOTES}

1.Je remercie particulièrement Myriam La Bruyère de m'avoir initié, il y a quelques années, à l'intéressante question de la réception de Haydn en France, à travers un remarquable mémoire de maîtrise sur l'édition parisienne des dernières symphonies du compositeur (Université de Rouen, Patrick Taïeb, dir.). Un grand merci également à Cécile Duflo pour avoir facilité mon travail de dépouillement de presse en me communiquant son index - encore inédit - de la Correspondance des Amateurs Musiciens (1803-1805).

2.Courrier de l'Europe, 26 mars 1810, « Grand concert, au bénéfice de M. Dussek ». 3.Lionel de LA LAURENCIE, «L'apparition des œuvres de Haydn à Paris ", Revue de musicologie, $\mathrm{n}^{\circ}$ 44, 1932, pp. 195-205. 
4.François Lesure, « Haydn en France », Bericht über die internationale Konferenz zum Andenken J. Haydns, Budapest, Akadémiai kiado, 1961, p. 80.

5.Les numéros de symphonies utilisés dans le présent article font référence au classement d'Anthony van Hoboken aujourd'hui couramment admis.

6.Johann Friedrich REICHARDT, Un Hiver à Paris sous le Consulat, Paris, Tallandier, 2003, p. 271, « 7 janvier 1803, second concert de la rue de Cléry ».

7.Ibid.

8.Concernant les seules symphonies parisiennes ( $n^{\circ} 82$ à 87 du catalogue Hoboken), on sait que l'ordre de composition fut une première fois inversé dans le catalogue de l'éditeur français Imbault $\left(n^{\circ} 83,87,85,82,86,84\right)$ puis à nouveau dans le catalogue de l'éditeur viennois Artaria, qui ne respecta pas les consignes de Haydn, celui-ci préconisant d'organiser la série de cette troisième manière : $\mathrm{n}^{\circ} 87,85,83,84,86,82$. 9.Courrier des Spectacles, 8 mars 1798.

10.Ibid., 11 juillet 1799.

11.Affiches, annonces et avis divers, 25 décembre 1791.

12.Ibid., 30 janvier 1792.

13.Thomas Betzwieser, «Zwischen Geschmackswandel und Genieästhetik :

Anmerkungen zur französischen Haydn-Rezeption im 18. Jahrhundert », dans Herbert SCHNEIDER, (dir.), Studien zu den deutsch-französischen Musikbeziehungen im 18. und 19.

Jahrhundert, Hildesheim, Olms, 2002, pp. 302-324 ; Jean GRIBENSKI, « Haydn », dans Marcelle BENOIT, (dir.), Dictionnaire de la musique en France aux XVIIe et XVIIIe siècles, Paris, Fayard, 1992, pp. 339-340 ; Lucie KAYAS, « Haydn », dans Joël-Marie FAUQUET, Dictionnaire de la musique en France au XIXe siècle, Paris, Fayard, 2003, pp. 583-584 ; Lionel de LA LAURENCIE, art. cit.

14.Courrier des Spectacles, 24 décembre 1800.

15.Journal de Paris, 15 mai 1779.

16.Correspondance des Amateurs Musiciens, 5 février 1803, « 5e Concert des Amateurs, rue de Cléry ».

17.Peut-être la Symphonie $n^{\circ} 94$ «La Surprise »? Mais plus probablement la Symphonie $n^{\circ}$ 100 « Militaire ».

18.Correspondance des Amateurs Musiciens, 26 février 1803, «7e concert de la rue de Cléry ».

19.Les symphonies « londoniennes » de Haydn se découpent en deux groupes de six œuvres composées pour les concerts du violoniste Salomon à Londres. Respectivement $n^{\circ} 93$ à 98 et nº 99 à 104.

20.Correspondance des Amateurs Musiciens, 23 février 1805, « 2e concert des Amateurs ».

21.Ibid., 30 mars 1805, « 6e concert des Amateurs ».

22.Ibid., 19 février 1803, « 6e Concert de la rue de Cléry ».

23.Ibid., 30 mars 1805, « 6e concert des Amateurs ».

24.Les clarinettes interviennent uniquement dans la seconde série des symphonies londoniennes ( $n^{\circ} 99$ à 104).

25.voir à ce propos Alexandre DRATWICKI, Un Nouveau Commerce de la virtuosité: métamorphoses de la symphonie concertante au sein des institutions musicales parisiennes (1780-1830), Thèse de l'Université de Paris IV-Sorbonne, 2003.

26.Voir par exemple les Affiches, annonces et avis divers des 8 et 10 avril 1792.

27.Feuille du jour, 13 novembre 1806.

28. Mercure de France, 24 décembre 1784, 4 et 23 avril 1785, 15 août 1785, 1er avril 1787. 29.Feuille du jour, 23 avril 1791, « Concert spirituel». 
30. Mercure de France, avril 1773.

31.Lionel de LA LAURENCIE, art. cit., p. 194.

32. Correspondance des professeurs et amateurs de musique, 5 janvier 1805.

33. Correspondance des Amateurs Musiciens, 25 février 1804, «Concert de la rue de Grenelle ».

34.Ibid.

35.Probablement $\mathrm{n}^{\circ} 44$ " Funèbre ", effectivement en mi mineur.

36. Correspondance des Amateurs Musiciens, 4 mars 1804, « 4e concert Cléry ».

37.Ibid., 12 février 1803, «6e concert des élèves du Conservatoire ».

38. Gazette de Paris, 22 avril 1813.

39.Journal de l'Empire, 21 mars 1811.

40.Journal des Débats, 10 octobre 1801.

41. Voir Affiches, annonces et avis divers des 20 et 22 avril 1791.

42.Voir Affiches, annonces et avis divers du 12 juin 1791.

43. "Samedi dernier a eu lieu le premier Concert de la rue de Cléry. On y a exécuté dans la perfection deux symphonies de Haydn. Je n'ai pu que renouveler, à cette occasion, le vœu que j'exprimais, il devrait venir à Paris, afin de connaître toute la perfection de ses œuvres. Nulle part on ne les joue comme ici. » (Johann Friedrich REICHARDT, op. cit., p. 253 [lettre de décembre 1802).

44.Correspondance des Amateurs Musiciens, 29 janvier 1803, « 4e concert des Amateurs, rue de Cléry ».

45.Par exemple le 5 octobre 1811 et le 2 octobre 1813.

46.Voir notamment les exemplaires mentionnés dans le Catalogue des fonds musicaux anciens en Ile-de-France, Préface de Jean Mongrédien, Ariam Île-de-France, 2003, tome 1, pp. 159-162.

47. Courrier des Spectacles, 1er septembre 1799.

48. "Bulletin de l'Assemblée nationale ", texte de la loi Le Chapelier, Gazette Nationale ou le Moniteur Universel, 15 janvier 1791.

49.Bibliothèque de la Comédie-Française : Dossier « Orchestre » / Documents comptables / Frais pour le ballet et l'orchestre 1755 - 1793 [cote : 2 AC] / « Copie et achat de musique 1753-1793 ». On y trouve la mention d'un achat de 1788, détaillé comme suit : « État des Simphonies achetées pour La Comédie Française l'année 1788. Les exemplaires sont doubles. »

50.« La musique instrumentale de Haydn est composée de symphonies de chambre à plus ou moins d'instruments, et de symphonies à grand orchestre, qu'à cause du grand nombre d'instruments nécessaires on ne peut guère jouer que dans un théâtre. ", (Henri Beyle dit STENDHAL, Haydn et Mozart, Paris, Michel de Maule, 2002, p. 57 lettre du 2 octobre 1808).

51.voir Annuaire dramatique, Paris, 1805.

52.Journal de Paris, 24 mars 1812.

53. Courrier des Spectacles, 14 janvier 1814. L'annonce précise encore : « L'ouverture, les airs de danse, les marches, sont de la composition de L. Moranges, et les ballets de M. Blondin. Ce drame a été représenté pour la première fois sur le théâtre de la Porte Saint-Martin, le 3 frimaire an 12 [25 novembre 1803]. »

54.Johann Friedrich REICHARDT, op. cit., p. 315.

55.Adaptation française de La Flûte enchantée de Mozart par Louis-Wenceslas Lachnith en 1801.

56.Courrier des Spectacles, 29 août 1801. 
57.François Lesure, « Haydn en France », Bericht über die internationale Konferenz zum Andenken J. Haydns, Budapest, Akadémiai kiado, 1961, p. 82. Ce mouvement lent de la Symphonie $n^{\circ} 53$ "L'Impériale », effectivement parodié dans Le Fat Dupé, est notamment repris par un certain « Mr Abraham » dans ses Dix petits airs pour deux clarinettes (Paris, Savigny, s.d.), non pas comme œuvre de Haydn, mais sous le titre « extrait du Fat dupé ».

58. Courrier des Spectacles, 10 avril 1802.

59. Correspondance des Amateurs Musiciens, 24 janvier 1804, « Concert de la rue de Grenelle ».

60.François Henri Joseph CASTIL-BLAZE, « Ballet », dans le Dictionnaire de Musique Moderne, Paris, Egron, 1821, p. 46. À l'article « Air » du même ouvrage (p. 24), on peut lire encore : « Les airs de danse ne sont plus calqués sur un modèle connu, le compositeur s'accorde avec le chorégraphe pour les formes, le caractère et l'extension qu'il convient de leur donner. Depuis le concerto jusqu'à la symphonie, depuis le finale jusqu'au vaudeville, toute musique vocale ou instrumentale peut s'unir à la danse ». 61. Courrier des Spectacles, 4 décembre 1798. Le même journal écrivait la veille : « Enfin l'opéra d'Alceste vient d'être orné de plusieurs pas de danse, d'une composition savante et délicieuse ; on les doit au cit. Gardel. Rien n'égale le goût et l'intelligence avec lesquels il a su faire sentir, par la variété des caractères de danse, les différentes parties de l'andante de la superbe symphonie en ut d'Haydn. Les contrastes de l'harmonie sont pour ainsi dire traduits avec une extrême délicatesse, et plus ce dessin sera connu, plus il sera goûté et apprécié. Ce morceau est habilement exécuté par les citoyennes Pérignon, Collomb, Chameroy, Louise et Milliere » (Courrier des Spectacles, 3 décembre 1798).

62.Voir Alexandre DRATWICKI, « Gossec et les premiers pas du ballet-pantomime français. Autour du succès de Mirza (1779) », livre-programme Grandes Journées Gossec, Versailles, Centre de Musique Baroque, 2002, pp. 101-116.

63. Courrier des Spectacles, 3 janvier 1800.

64.Ibid., 29 août 1801.

65.Les ballets suivants n'ont pas tous prêtés à un dépouillement systématique, mais contiennent de façon certaine au moins un extrait de symphonie de Haydn : Psyché de Miller et Gardel (1790), Télémaque dans l'île de Calypso de Miller et Gardel (1790), Le Jugement de Pâris de Méhul et Gardel (1793), Héro et Léandre de Lefebvre et Milon (1799), Daphnis et Pandrose de Méhul et Gardel (1803), Achille à Scyros de Cherubini et Gardel (1804), Alexandre chez Apelles de Catel et Gardel (1808), Persée et Andromède de Méhul et Gardel (1810), L'Enfant Prodigue de Berton et Gardel (1811), Mars et Vénus de Schneitzhoeffer et Blache (1826) (Voir Marian SMITH, Ballet and Opera in the Age of Giselle, Princeton University Press, 2000, pp. 104-107).

66.Johann Friedrich REICHARDT, op.cit., p. 76 (commentaire du Jugement de Pâris de Méhul et Gardel).

67.Nicolas-Étienne FRAMERY, Pierre-Louis GINGUÉNÉ et Jérôme-Joseph de MOMIGNY, « Symphonie ", dans l'Encyclopédie méthodique, Paris, 1818, t.II, p. 412.

68.STENDHAL, Haydn et Mozart, Paris, Michel de Maule, 2002, p. 59.

69.Voir Alexandre DRATWICKI, « Haydn et Mozart dansés à l'Opéra de Paris : le transfert émotionnel entre danse et musique dans le ballet-pantomime classique (1776-1815) ", dans De la Rhétorique des passions à l'expression du sentiment, Paris, Cité de la Musique, 2003, pp. 78-87. 
70.André Modeste GRETRY, Mémoires ou Essais sur la musique, Paris, Imprimerie de la République, 1/1789, 1797, t.I, p. 244.

71.BAILLOT, LEVASSEUR, CATEL et BAUDIOT, Méthode de violoncelle et de basse d'accompagnement adoptée par le Conservatoire Impérial de Musique pour servir à l'étude dans cet établissement, Paris, Mme Le Roy, s.d, préface.

72.André Modeste GRETRY, op. cit., t. I, p. 347.

73. Correspondance des Amateurs Musiciens, 4 janvier 1804.

74.Les écrits de certains théoriciens n'omettent pas de signaler les qualités techniques des ouvrages de Haydn, mais sans pour autant taire leur qualité « vocale », voire « dramatique » : «Haydn, dans ses symphonies, est un véritable modèle pour toutes les parties de l'art musical. Du motif le plus simple et souvent le plus commun, il fait sortir le chant le plus élégant, le plus majestueux. Ses sujets sont toujours clairement exposés, habilement développés; et chaque morceau offre un poème en action, auquel il ne manque que la parole. L'emploi des instruments à vent y est admirable », (Alexandre CHORON et François FAYOLLE, « Haydn », dans Dictionnaire historique des musiciens, Paris, Valade, 1810, t. I, p. 322).

75.Correspondance des Amateurs Musiciens, 11 février 1804, «Concert de la rue de Grenelle ».

76.Giovanni Giuseppe CAMBINI, Nouvelle méthode théorique et pratique pour le violon, Paris, Naderman, 1800.

77.Ibid.

78.Ibid.

79.Yves GÉRARD, « Musique instrumentale et expression : de l'imitation contestée à l'autonomie rêvée. ", dans Actes du colloque De la Rhétorique des passions à l'expression du sentiment, Paris, Cité de la Musique, 2003, p. 111.

80. Albert Christophe DIES, Informations biographiques sur Joseph Haydn, Vienne Camesina, 1810, dans Joseph HAYDN, Autobiographie. Premières biographies, traduites et présentées par Marc VIGNAL, Paris, Flammarion, 1997, p. 250.

81.Voir les exemplaires repérés dans le Catalogue des fonds musicaux anciens en Île-deFrance, op. cit., pp. 162-163.

82.STENDHAL, op. cit., p. 59.

\section{RÉSUMÉS}

La postérité a retenu de l'époque classique les trois figures musicales emblématiques que sont Haydn, Mozart et Beethoven en reléguant souvent le premier à l'ombre des deux autres. Or, entre 1780 et 1820, Joseph Haydn connut en Europe - en France tout particulièrement - un engouement qui dépassa de loin ceux de ses deux grands contemporains. Plus que des ouvrages épisodiques comme La Création ou le Stabat Mater (censé rivaliser avec celui de Pergolèse), ses symphonies témoignent d'un succès bien curieux: destinées aux concerts, elles furent progressivement utilisées dans des cadres artistiques aussi variés que les entractes de la Comédie Française, les bals de la Cour impériale ou les préludes de certaines tragédies lyriques. Ultime "dramatisation » de ce répertoire symphonique, l'utilisation de mouvements détachés comme partie intégrante 
des ballets-pantomimes modernes de l'Opéra de Paris confère à cette musique un potentiel expressif qu'elle ne revendiquait pas à l'origine.

The reception of Haydn's symphonies in Paris. While Haydn, Mozart, and Beethoven are considered as the three emblematic musical figures of the Classical era, posterity has often relegated Haydn to the shadow of the other two. However, between 1780 and 1820, Joseph Haydn enjoyed in Europe - and France in particular - a cult following which far surpassed those of his two great contemporaries. His symphonies - even more than the occasional works such as The Creation or the Stabat Mater (supposedly to rival that of Pergolesi) - witnessed a curious success: destined for the concert hall, these works became progressively used in artistic contexts as varied as the entractes of the Comédie Française, the bals of the Imperial Court, or the preludes of some tragédies lyriques. The ultimate dramatization of this symphonic repertoire and the use of the individual movements as an integral part of the modern ballet-pantomimes of the Paris Opéra bestows upon this music an expressive potential which removes it far from its original conception.

\section{INDEX}

Mots-clés : musique, Haydn (Joseph), symphonie, ballet, réception, Empire

\section{AUTEUR}

\section{ALEXANDRE DRATWICKI}

Docteur en musicologie, ancien pensionnaire de l'Académie de France à Rome, Centre de recherche "Langages musicaux", Université de Paris IV-Sorbonne, 187 avenue de Clichy, 75017 Paris 